\section{Evaluating Antiretroviral Therapy Initiation in HIV-Associated Malignancy: Is There Enough Evidence to Inform Clinical Guidelines?}

\author{
Linda N. Oseso, MPH; Elizabeth Y. Chiao, MD, MPH; \\ and Rachel A. Bender Ignacio, MD, MPH
}

For HIV-associated malignancies (HIVAMs), limited high-quality data are available to support specific timing of antiretroviral therapy (ART) initiation for ART-naïve persons who require chemotherapy. HIVAMs include 3 AIDS-defining cancers (ADCs; Kaposi sarcoma [KS], non-Hodgkin lymphoma [NHL], and cervical cancer) and non-aids-defining cancers (NADCs; all other tumors). HIVAMs are a highly heterogeneous group of diseases with variable linkage to immunosuppression, viral oncogenes, and other risk factors. ART initiation may frequently be delayed in ARTnaïve persons with HIVAMs due to concerns about immune reconstitution inflammatory syndrome (IRIS), complex drug-drug interactions (DDIs), treatment burden, and challenges associated with cotreatment of diseases that traditionally have been managed separately. ${ }^{1-3}$

The timing of ART initiation in HIVAMs has changed drastically over the years. In the 1990s and early 2000s, ART was often held or not started in persons undergoing chemotherapy. ${ }^{4}$ Now that modern ART and cancer treatment options make ART timing and DDIs less complex, ART initiation during cancer therapy may be less problematic in the United States. However, in sub-Saharan Africa, where the largest burden of HIVAM exists, cytotoxic chemotherapy and older ART are the norm, and care for the 2 diseases are rarely integrated in the same institution; thus, concurrent treatment of both diseases is challenging.

Overall, it is clear that early initiation of ART in persons with HIV should be provided as soon as possible after HIV diagnosis, regardless of CD4+ T-cell count (CD4 count). Two large randomized trials published in 2015 confirmed a survival benefit for immediate ART in persons not previously meeting criteria for ART,5,6 and subsequent WHO guidelines immediately aligned with this strategy. ${ }^{7}$ Not addressed in these guidelines is when to start ART in the presence of serious opportunistic infection (OI) or other disease that might make strategic short-term delay of ART initiation appropriate. Clinical trials for cryptococcal meningitis and tuberculosis (TB) coinfections support short delays in ART initiation (2-10 weeks) after start of OI treatment to balance risks of IRIS, toxicities, and death; overall, these studies favor avoiding prolonged delay. ${ }^{8-12}$ The risk of IRIS is highest in infections or malignancies with a strong inflammatory component, in persons with low CD4 count, and in those with a short interval between initiation of OI treatment and ART. IRIS is rarely reported in solid tumors, but is common in KS and lymphoma. ${ }^{13-15}$

Although HIVAMs in North America and Europe are often comanaged by specialists, including specialty pharmacists, care for HIVAM in low-resource settings is further complicated by reliance on more cytotoxic chemotherapy regimens, fewer available ART regimen choices, and limited ability to monitor for and manage adverse events. Given the increasing burden of HIVAMs in low-resource settings and the overwhelming evidence that coordinated HIV/TB care has improved outcomes related to similar hurdles, ${ }^{16}$ clinical guidelines such as the NCCN Guidelines for Cancer in People Living With HIV (in this issue), can inform initiation and management of ART in persons with comorbid cancer. ${ }^{17}$

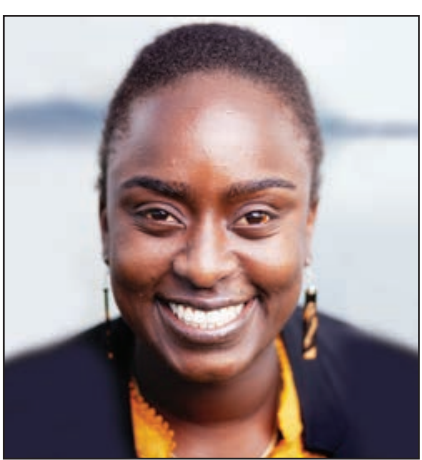

Linda N. Oseso, MPH

Linda N. Oseso, MPH, is a Project Manager at the HIV Vaccine Trials Network (HVTN) based at the Fred Hutchinson Cancer Research Center. She received her bachelor's degree from the University of California, Berkeley and completed her MPH in Epidemiology at the University of Washington. Ms. Oseso's research interests are in global health, HIV/ sexually transmitted infections, mental health, adolescent health, community-based participatory research, and improving health access via culturally appropriate public health solutions. 


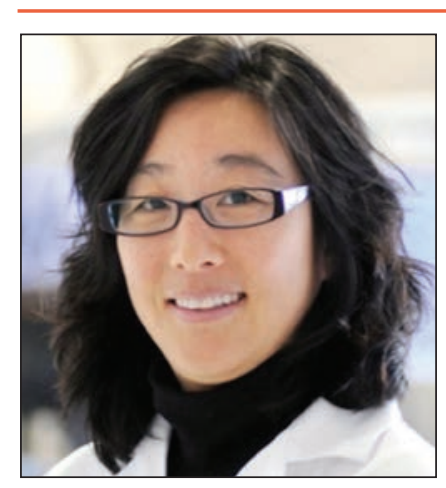

Elizabeth Y. Chiao, MD, MPH

Elizabeth Y. Chiao, MD, MPH, is an Associate Professor of Medicine in the Infectious Diseases Division at Baylor College of Medicine. She obtained her MD at Cornell University and her MPH in Epidemiology at Yale University. She completed residency in internal medicine at the University of Utah, and is double-boarded in infectious diseases (Baylor) and medical oncology (Memorial Sloan Kettering Cancer Center). Dr. Chiao's research interests include the study of virally associated malignancies, particularly HPV-associated cancers, and the prevention, diagnosis, and treatment of HIV-associated cancers. She conducts studies with the AIDS Clinical Trials Group and AIDS Malignancy Consortium.

\section{When Do HIVAM Experts Start ART?}

No interventional or observational trials were noted, outside of those in KS, that answer the question when to start ART. ${ }^{18}$ Therefore, the authors leveraged the AIDS Malignancy Consortium (AMC; a clinical trials group supported by NCI) to survey expert clinicians who treat HIVAM to assess whether consensus exists on timing of ART initiation in common cancers, and consequently, sufficient equipoise to support clinical trials to answer this question for different cancers. AMC collaborators also offered email addresses of non-AMC clinicians in the United States and Africa with expertise in HIVAM. The survey was administered from November 2015 through January 2016 and was logged anonymously; neither survey responses nor lack of survey completion could be traced back to individual email addresses. This survey was considered exempt from ethics review by the Seattle Cancer Consortium Institutional Review Board. The AMC approved this survey (supplemental eAppendix 1, available with this article at JNCCN.org) and facilitated its distribution to AMC members. Expert clinicians were asked about ART initiation in ART-naïve patients with KS, breast cancer, lung cancer, NHL, Hodgkin lymphoma, gastrointestinal tumors (adenocarcinoma, squamous cell carcinoma), or cervical cancer requiring chemotherapy.

A total of 28 expert clinicians of approximately 90 that were contacted $(\approx 31 \%)$ provided input on their standard of practice. Most responding clinicians were oncologists $(57 \%)$ or infectious disease (ID) specialists $(29 \%)$ and from academic institutions (90\%); 60\% were based in the United States and the remainder practiced in 6 African countries.

Clinicians did not differentiate ideal timing for ART initiation when considering ADCs versus NADCs $(P=.96$; Table 1$)$. Timing of ART initiation for ADCs or NADCs was not different by continent of clinical practice. Considering all cancers together, there was a trend toward US clinicians more frequently recommending ART initiation before or concurrent with chemotherapy start compared with their African colleagues $(P=.09)$. Clinical specialty impacted the recommended timing of ART initiation overall $(P=.004)$ and for ADCs $(P=.05)$, but was not significant for NADCs $(P=.08)$; ID physicians were more likely to recommend ART initiation as soon as possible regardless of chemotherapy start, whereas oncologists were more likely to stagger ART before or after initiation of chemotherapy, but not start both concurrently. Clinicians uncommonly reported using CD4 count to guide ART timing but would do so more frequently for NADCs than ADCs ( $28 \%$ vs $16 \%$, data not shown; $P=.05$ ).

\section{KS-Specific Considerations}

For KS, more than half of clinicians favored starting ART before chemotherapy and $20 \%$ favored concurrent administration, but several commented that ART timing should be considered differently depending on disease stage. Some consider chemotherapy first for up to 2 cycles before ART initiation for patients with visceral tumor involvement or extensive disease to prevent IRIS. Some clinicians specified that ART should be either started first or used exclusively (vs in combination with chemotherapy) for limited KS disease. For those who recommended ART before chemotherapy, the median interval from ART initiation to chemotherapy start was 4 weeks (interquartile range, 3-9 weeks). Factors considered for ART timing included KS stage (70\%), concurrent OIs (75\%), possibility of DDIs (50\%), and IRIS (85\%) (Table 1).

\section{Other Cancers}

ART-initiation strategy as reported by clinicians surveyed was similar for the other 6 cancers aside from KS (Table 1). More than half of clinicians start ART either concurrently or before chemotherapy (range, 64\%-84\%), with a median of 2 weeks before chemotherapy when suggesting staggered initiation. Up to $50 \%$ of responding clinicians remained concerned about IRIS when starting ART, including for solid tumors. 
A minority of clinicians stated they would allow some delay of ART for CD4 count $\geq 500$ cells $/ \mathrm{mcL}$, but no respondent recommended not initiating ART within 2 months of first chemotherapy.

\section{Openness to A Randomized Clinical Trial Varying Timing of ART Initiation}

Depending on tumor type, between half and three-quarters of clinicians would consider enrolling patients similar to those discussed in our survey in a trial in which ART was started immediately or delayed until up to 2 cycles of chemotherapy were completed. There was no difference in openness to clinical trial referral or participation with respect to the cancer type in question.

\section{From Opinion to Action}

Most expert clinicians surveyed would begin ART either before or concurrent with cytotoxic chemotherapy for most HIVAMs. A difference in treatment pattern was seen between oncologists and ID specialists, with ID clinicians more strongly in favor of starting ART as soon as possible. This may reflect a difference in perceived risk of untreated HIV versus complexity and toxicities of ART with chemotherapy. US-based clinicians also tended to recommend ART earlier than African clinicians. Although not statistically significant, this observation possibly reflects higher comfort with starting ART if less toxic regimens and closer monitoring are available. No difference was found in preferred ART timing based on whether the cancer was AIDS-defining or non-AIDS-defining, despite the fact that historically the presence of an ADC was a standalone clinical indication for ART. Given the somewhat artificial separation between ADCs and NADCs, we were relieved that this historical practice was not perpetuated, at least in these responses. Clinical trials, particularly in non-AIDS-defining solid tumors and targeted specifically to resource-limited versus resource-replete settings, could augment the available limited observational data to support these guidelines recommendations, especially for resource-limited settings.

Several recent studies have elucidated some chemotherapy/ART DDIs that require consideration, including several combinations that should be avoided based on CYP3A4 interaction (eg, protease inhibitors with docetaxel). ${ }^{19-23}$ Several experts therefore cite the need for prospective studies to further direct chemotherapy dosing, such that toxicities, especially bone marrow suppression, can be minimized without delivering subtherapeutic chemotherapy or risking development of HIV resistance. ${ }^{24}$

The best data on chemotherapy and concurrent ART relates to NHL, for which several studies have shown that administering ART is associated with higher rates of complete response and longer overall survival, albeit with worsened hematologic toxicities, resulting in more frequent dependence on blood products and some risk of reversing viral suppression. ${ }^{25-28}$ Whether increased reliance on granulocyte colonystimulating factor or blood products would diminish the survival benefit or make concurrent ART initiation less feasible in resource-limited settings is not clear. To date, most studies have been performed in higher-resource settings and have little applicability to sub-Saharan Africa other than to offer caution in prescribing certain regimens concurrently. Additionally, these studies evaluated the binary state of being on or not on ART during the course of chemotherapy, and lumped together previously ART-naïve patients with those who had ART interrupted to receive chemotherapy. These studies were performed before publication of WHO recommendations that all HIV-infected persons receive ART, and could not account for inherent differences

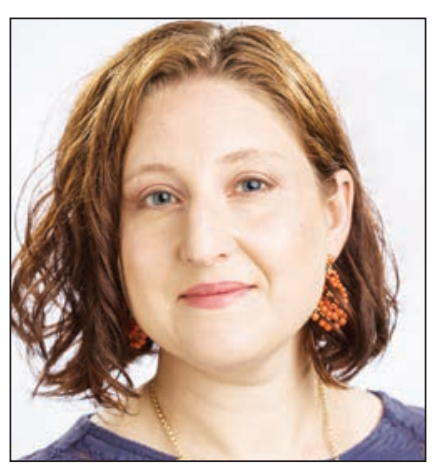

Rachel A. Bender Ignacio, $\mathrm{MD}, \mathrm{MPH}$

Rachel A. Bender Ignacio, MD, $\mathrm{MPH}$, is an Assistant Professor of Medicine in the Division of Allergy and Infectious Diseases at the University of Washington (UW) and is an Associate in the Vaccine and Infectious Diseases Division at Fred Hutchinson Cancer Research Center. She studied medicine, global health, and epidemiology at UW, completed residency in internal medicine at Massachusetts General Hospital/ Harvard, and completed a fellowship in infectious diseases at UW. She is a member of the AIDS Malignancy Consortium and her research focuses on outcomes associated with viral coinfections and inflammation in HIV. 


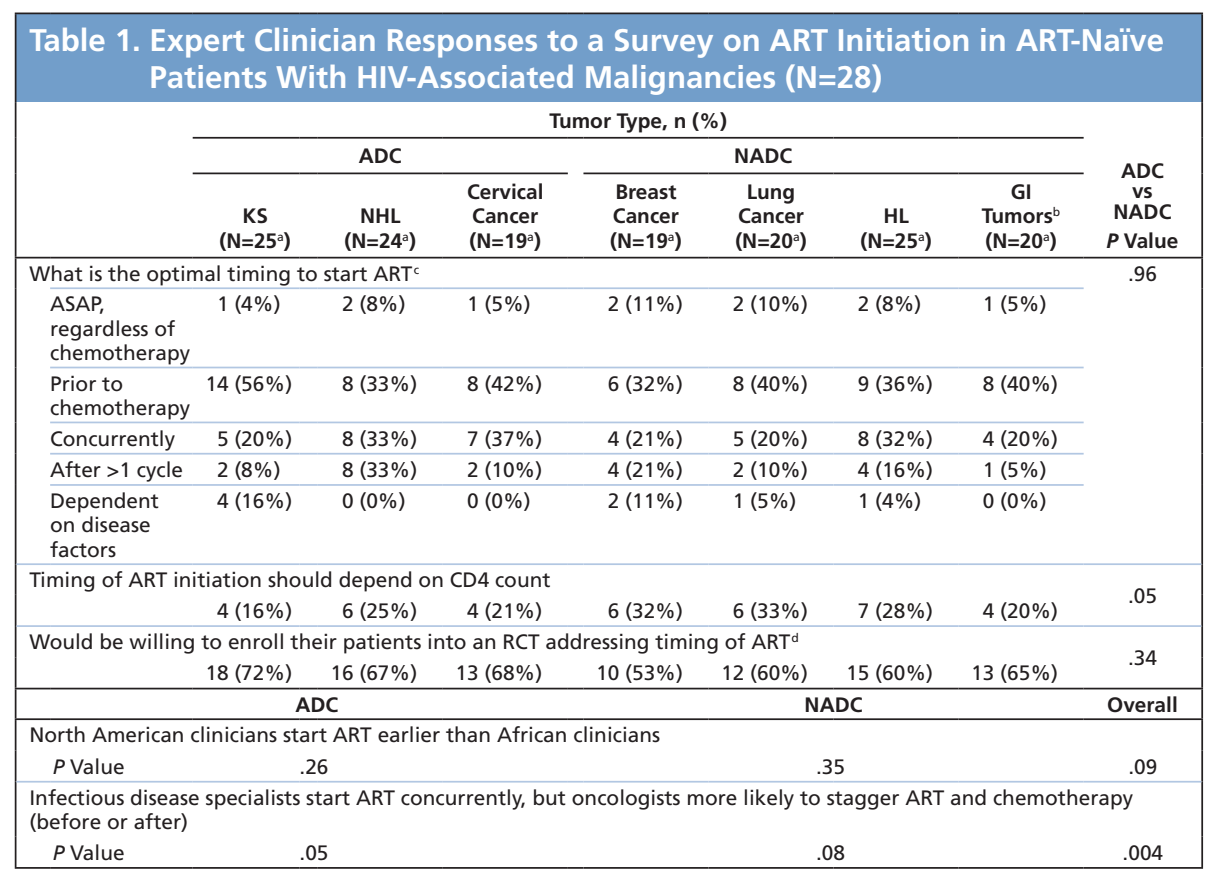

Abbreviations: ADC, AIDS-defining cancer; ART, antiretroviral therapy for HIV; CD4, CD4+ T-cell count; GI, gastrointestinal; HL, Hodgkin lymphoma; KS, Kaposi sarcoma; NADC, non-AIDS-defining cancer; NHL, non-Hodgkin lymphoma; RCT, randomized controlled trial.

${ }^{a}$ Maximum number of clinicians who responded to questions regarding each tumor type. Some clinicians who reported not treating a particular cancer did not complete the questions pertaining to that cancer. Missing values are not included. ${ }^{b}$ Clinicians were requested to consider squamous cell and adenocarcinomas of the Gl tract.

"Clinicians were requested to consider squamous cell and adenocarcin

${ }^{\mathrm{d}} \mathrm{RCT}$ proposed in survey randomized persons with each HIV-associated malignancy to start ART immediately (before or concurrent to chemotherapy initiation) vs waiting for up to 2 cycles of chemotherapy to initiate ART.

between patient groups. These studies did not directly address the question of when an ART-naïve patient should start ART with respect to cancer treatment.

Based on the responses of the expert clinicians surveyed, opinion regarding the timing of ART initiation was varied. Although most clinicians supported starting ART as soon as feasible barring a specific rationale for delaying ART, such as DDIs or IRIS, clear evidence regarding the timing of ART initiation for specific cancers is lacking. Many of our colleagues support an effectiveness study of standard chemotherapy regimens plus immediate versus minimally delayed ART initiation. One proposed study design would be to randomize ART-naïve persons with upper aerodigestive tumors (head and neck, lung, esophagus, gastric cancers) in cancer centers in sub-Saharan Africa to start a standard ART regimen concurrently with chemotherapy or wait until after 2 cycles of chemotherapy. We would consider including only persons with CD4 >100 cells/mcL to avoid delaying chemotherapy for persons who are extraordinarily immunosuppressed; this was the population at highest risk of death with delayed ART in similar studies in persons with TB or other serious OIs. ${ }^{10-12}$ These types of studies may be particularly useful in resource-limited settings to define the risks and benefits of these strategies, especially with respect to adverse drug events and neutropenic or opportunistic infections. The results of such trials would support these guidelines recommendations, coordinating ART initiation and cancer care. In the absence or in advance of results of a future randomized trial dedicated to answering this question, we hope that the NCCN Guidelines for Cancer in People Living With HIV, which are based on expert opinion and available data for each cancer type and clinical setting, can assist clinicians, especially those in sub-Saharan Africa, to initiate and continue ART during HIVAM treatment. We hope that the pursuit of data and the 
utilization of the proposed clinical practice guidelines reap similar benefits to those that have resulted from tackling similar questions in HIV/TB coinfection.

\section{Acknowledgments}

The authors wish to thank Dr. Ron Mitsuyasu for facilitating this work and reviewing the manuscript, and the AMC for cooperating with this project, including distributing the survey to members.

\section{References}

1. Deeken JF, Tjen-A-Looi A, Rudek MA, et al. The rising challenge of non-AIDS-defining cancers in HIVinfected patients. Clin Infect Dis 2012;55:1228-1235.

2. Bateganya MH, Stanaway J, Brentlinger PE, et al. Predictors of survival after a diagnosis of non-Hodgkin's lymphoma in a resource-limited setting: a retrospective study on the impact of HIV infection and its treatment. J Acquir Immune Defic Syndr 2011;56:312-319.

3. Aboulafia DM. Decision making in non-AIDS-defining malignancies. Lancet Oncol 2012;13:1172-1173.

4. Little RF, Pittaluga S, Grant N, et al. Highly effective treatment of acquired immunodeficiency syndrome-related lymphoma with dose-adjusted EPOCH: impact of antiretroviral therapy suspension and tumor biology. Blood 2003;101:4653-4659.

5. Lundgren JD, Babiker AG, Gordin F, et al. Initiation of antiretroviral therapy in early asymptomatic HIV infection. N Engl J Med 2015;373:795-807.

6. Danel C, Moh R, Gabillard D, et al. A trial of early antiretrovirals and isoniazid preventive therapy in Africa. N Engl J Med 2015;373:808-822.

7. WHO. Guideline on When to Start Antiretroviral Therapy and on Pre-Exposure Prophylaxis for HIV. Available at: http://apps.who.int/iris/bitstream/handle/10665/186275/9789241509565_eng.pdf;jsessionid=4A03C127AA38 7AE9B39F4DF9DACDF32E?sequence=1. Accessed July 2, 2018.

8. Boulware DR, Meya DB, Muzoora C, et al. Timing of antiretroviral therapy after diagnosis of cryptococcal meningitis. N Engl J Med 2014;370:2487-2498.

9. Makadzange AT, Ndhlovu CE, Takarinda K, et al. Early versus delayed initiation of antiretroviral therapy for concurrent HIV infection and cryptococcal meningitis in sub-Saharan Africa. Clin Infect Dis 2010;50:15321538.

10. Havlir DV, Kendall MA, Ive P, et al. Timing of antiretroviral therapy for HIV-1 infection and tuberculosis. N Engl J Med 2011;365:1482-1491.

11. Török ME, Yen NT, Chau TT, et al. Timing of initiation of antiretroviral therapy in human immunodeficiency virus (HIV)-associated tuberculous meningitis. Clin Infect Dis 2011;52:1374-1383.

12. Zolopa A, Andersen J, Powderly W, et al. Early antiretroviral therapy reduces AIDS progression/death in individuals with acute opportunistic infections: a multicenter randomized strategy trial. PLoS One 2009;4:e5575.

13. Letang E, Lewis JJ, Bower $M$, et al. Immune reconstitution inflammatory syndrome associated with Kaposi sarcoma: higher incidence and mortality in Africa than in the UK. AIDS 2013;27:1603-1613.

14. Achenbach CJ, Harrington RD, Dhanireddy S, et al. Paradoxical immune reconstitution inflammatory syndrome in HIV-infected patients treated with combination antiretroviral therapy after AIDS-defining opportunistic infection. Clin Infect Dis 2012;54:424-433.

15. Gopal S, Patel MR, Achenbach CJ, et al. Lymphoma immune reconstitution inflammatory syndrome in the center for AIDS research network of integrated clinical systems cohort. Clin Infect Dis 2014;59:279-286.

16. World Health Organization. WHO policy on collaborative TB/HIV activities: guidelines for national programmes and other stakeholders. Geneva, Switzerland: World Health Organization; 2012.

17. Adebamowo $\mathrm{CA}$, Casper $\mathrm{C}$, Bhatia $\mathrm{K}$, et al. Challenges in the detection, prevention, and treatment of HIV-associated malignancies in low- and middle-income countries in Africa. J Acquir Immune Defic Syndr 2014;67(Suppl 1):S17-26.

18. Mosam A, Shaik F, Uldrick TS, et al. A randomized controlled trial of highly active antiretroviral therapy versus highly active antiretroviral therapy and chemotherapy in therapy-naive patients with HIV-associated Kaposi sarcoma in South Africa. J Acquir Immune Defic Syndr 2012;60:150-157.

19. Berretta M, Caraglia M, Martellotta F, et al. Drug-drug interactions based on pharmacogenetic profile between highly active antiretroviral therapy and antiblastic chemotherapy in cancer patients with HIV infection. Front Pharmacol 2016;7:71.

20. Rudek MA, Chang CY, Steadman K, et al. Combination antiretroviral therapy (cART) component ritonavir significantly alters docetaxel exposure. Cancer Chemother Pharmacol 2014;73:729-736.

21. Pillai VC, Parise RA, Christner SM, et al. Potential interactions between HIV drugs, ritonavir and efavirenz and anticancer drug, nilotinib - a study in primary cultures of human hepatocytes that is applicable to HIV patients with cancer. J Clin Pharmacol 2014;54:1272-1279.

22. Torres HA, Rallapalli V, Saxena A, et al. Efficacy and safety of antiretrovirals in HIV-infected patients with cancer. Clin Microbiol Infect 2014;20:O672-679.

23. Casado JL, Machuca I, Banon S, et al. Raltegravir plus two nucleoside analogues as combination antiretroviral therapy in HIV-infected patients who require cancer chemotherapy. Antivir Ther 2015;20:773-777.

24. Torres HA, Mulanovich V. Management of HIV infection in patients with cancer receiving chemotherapy. Clin Infect Dis 2014;59:106-114. 
Oseso et al

25. Barta SK, Xue X, Wang D, et al. Treatment factors affecting outcomes in HIV-associated non-Hodgkin lymphomas: a pooled analysis of 1546 patients. Blood 2013;122:3251-3262.

26. Simonelli C, Zanussi S, Cinelli R, et al. Impact of concomitant antiblastic chemotherapy and highly active antiretroviral therapy on human immunodeficiency virus (HIV) viremia and genotyping in HIV-infected patients with non-Hodgkin lymphoma. Clin Infect Dis 2003;37:820-827.

27. Vaccher E, Spina M, di Gennaro G, et al. Concomitant cyclophosphamide, doxorubicin, vincristine, and prednisone chemotherapy plus highly active antiretroviral therapy in patients with human immunodeficiency virus-related, non-Hodgkin lymphoma. Cancer 2001;91:155-163.

28. Weiss R, Mitrou P, Arasteh K, et al. Acquired immunodeficiency syndrome-related lymphoma: simultaneous treatment with combined cyclophosphamide, doxorubicin, vincristine, and prednisone chemotherapy and highly active antiretroviral therapy is safe and improves survival-results of the German Multicenter Trial. Cancer 2006;106:1560-1568. 